\title{
Utilização do Conceito de Conjuntos Nebulosos na Otimização de Ferramentas de Priorização em Ergonomia: o caso da Matriz SIC-Fuzzy
}

\author{
Use of Fuzzy Logics for the Optimization of Ergonomics Prioritizing \\ Tools: The Case of SIC/Fuzzy Matrix
}

\author{
1 Marcello Silva e Santos marcellosanto@hotmail.com \\ 2 Claudio Henrique Santos Grecco \\ ${ }^{3}$ Mario Cesar Rodriguez Vidal
}

\footnotetext{
1 Centro Universitário de Volta Redonda, UniFOA.

2 Instituto de Engenharia Nuclear, IEN.

3 Universidade Federal do Rio de Janeiro, UFRJ.
}

\section{RESUMO}

A matriz de Priorização tem sido uma das ferramentas mais frequentes para auxiliar nas tomadas de decisões relacionadas a projetos. Por sua vez, as ações ergonômicas resultam em múltiplas necessidades de enquadramento, sendo algumas delas mais urgentes e necessárias, ou ainda outras complexas, que exijam ações de engenharia e, portanto, tendem a demorar a serem implementadas. Assim, matrizes de priorização possibilitariam eleger, por critérios diferenciados, os fatores de riscos e a urgência de cada problema que demanda uma determinada ação. A Matriz SIC/Fuzzy é uma evolução da Matriz de Priorização SIC ${ }^{\circledR}$, ferramenta desenvolvida pelo laboratório GENTE/Fuzzy da UFRJ. Ela relaciona os itens a serem analisados por critérios de saúde e segurança ocupacional, sendo utilizada no processo de tomada de decisão em ergonomia. O índice "SIC" permite, portanto, determinar o grau de gravidade ocupacional de cada posto de trabalho. A apropriação da Lógica Fuzzy à ferramenta original incorpora variáveis que representam atributos cognitivos humanos, transformando-os em um formato numérico que pode ser quantificado e mensurado. A teoria fuzzy é usada, essencialmente, para mapear modelos quantitativos de tomada de decisão e em métodos de representação em ambientes de incertezas e imprecisões. Neste contexto, podese utilizá-la juntamente com indicadores de priorização por criticidade utilizados em Ergonomia. $O$ trabalho descreve 0 modelo e apresenta experiências iniciais de sua aplicação.

\section{PALAVRAS-CHAVE}

lógica fuzzy; ferramentas de gestão; fisiologia; ergonomia.

\begin{abstract}
Prioritizing Tools are the most frequently used instruments to help Project Management related to decision making. On the other hand, ergonomic actions result in a multitude of compliance requirements, some more urgent and needed than others, let alone complexity issues that may require some engineering action, therefore being more difficult and time-demanding to implement. Thus, Priority Matrices allow for selecting, by different criteria, risk and urgency factors for each identified problem, preempting further action. The SIC/ Fuzzy Matrix is an earlier version of a Prioritizing Matrix (SIC@), an instrument conceived by the GENTE Lab, at the Federal University of Rio de Janeiro. It sorts out issues in need of being evaluated by health and occupational safety aspects, therefore fit to be applied in the decision making process of Ergonomics Projects. The SIC Index helps to determine each workstation's occupational severity level, in terms of fitness, comfort, hazards and other issues. The appropriation of Fuzzy Logics to the original instrument allows the embedment of variables that better represent human cognitive attributes, turning them into a numeric format that become quantifiable and measurable. Fuzzy Logics is essentially used in mapping quantitative models for decision making and representation methods in imprecise and uncertain environments. In this context, it is possible to combine its use in severity control processes, common in Ergonomic actions. This paper describes the model and presents initial experiences and some findings related to its application.
\end{abstract}

\section{KEYWORDS}

fuzzy logics; management tools; physiology; ergonomics.

\section{Como você deve citar?}

SILVA e SANTOS, Marcello; GRECCO, Claudio Henrique Santos; VIDAL, Mario Cesar Rodriguez. Utilização do Conceito de Conjuntos Nebulosos na Otimização de Ferramentas de Priorização em Ergonomia: o caso da Matriz SIC-Fuzzy. Cadernos UniFOA, Volta Redonda, n. 29, p. 33-47, dez. 2015. 


\section{INTRODUÇÃo}

Os mecanismos do processo decisório e a tomada de decisão em si envolvem o processo de triagem e seleção de alternativas, sendo uma das mais importantes características do processo de gestão. Entretanto, essa escala de importância envolve variáveis de análise dependentes e independentes. Em outras palavras, se a escolha possui caráter econômico, critérios de valor podem ser posicionados acima de classes de riscos, por exemplo. Por outro lado, se o indicador de referência for o risco - ou a prevenção do mesmo - como no caso de uma intervenção ergonômica, então o risco em si passa a ser um "valor" a ser considerado. No caso do estudo em tela, a etapa de priorização foi subsequente à etapa de observações e serviu principalmente para a determinação do índice de severidade ocupacional dos postos de trabalho selecionados pelo departamento envolvido.

O estudo visa demonstrar que a lógica fuzzy pode incorporar às matrizes de decisão utilizadas em Ergonomia um mecanismo de aproximação à realidade, já que atribui valores equivalentes ao juízo de razão de acordo com atributos pré-selecionados. Isso minimiza os efeitos adversos da aplicação de questionários, listas de verificação e outros instrumentos de coleta de dados que precisam ser aplicados por diferentes profissionais, com formações diferentes e em contextos diversos. Ao contrário do pensamento dominante, a lógica fuzzy nesse caso estaria melhor introduzindo o fator humano nos processos de tomada de decisão. Esse artigo não esgota o tema, porém objetiva propor discussões e reflexões que ajudem a aprimorar os mecanismos de tomada de decisão.

A matriz SIC/Fuzzy pode ser utilizada em diferentes situações, mas seu desenvolvimento se consolidou no processo de aplicação de um método de determinação de tempos de recomposição de fadiga denominado RFad, desenvolvido pelo Laboratório GENTE/Fuzzy, vinculado ao COPPE/UFRJ. 0 método RFad busca responder no plano operacional às necessidades de adequação normativa e meIhoria de indicadores de saúde ocupacional, características importantes dos processos de apreciação e análise ergonômica. Ainda que não seja o objetivo deste artigo abordar detalhadamente o método, é importante situar o contexto em que a matriz foi construída, facilitando a identificação de seus aspectos positivos e aplicabilidade.

\section{DESENVOLVIMENTO DO MODELO}

O modelo de priorização em questão consolidou-se a partir de um projeto desenvolvido em uma indústria do setor automotivo. Na empresa em questão foi realizada uma análise do processo de trabalho, com ênfase na ergonomia da atividade, em 45 postos de trabalho, combinando ferramentas de análise de processos com a Ergonomia e ferramentas de processo, além de um modelo de priorização, ao qual seria então incorporado a teoria dos conjuntos fuzzy. 0 Quadro 1 a seguir mostra um exemplo do cálculo de um sistema de indexadores SIC, combinando escalas de gravidade, urgência e tendência (GUT), que nos estudos iniciais não incorporava a lógica fuzzy, às anotações de enquadramento normativo e desconformidade, em uma atividade de trabalho considerada crítica.

De acordo com esta indicação, medidas de reformulação e treinamento, à luz da avaliação realizada, devem ser encetadas sob indicativo de urgência determinado pelo índice e caracterizado pela codificação de cores que facilita a identificação dentre os múltiplos resultados em uma matriz de tabulação. Quanto mais postos entram na composição, mais significativo é o emprego da ferramenta e seus recursos. 
Quadro 1 - Exemplo de cálculo do Indicador SIC

\begin{tabular}{|c|c|c|c|c|c|c|}
\hline Posto & Constrangimentos & Ação & Enquadramento & Desconformidades & GUT & SIC \\
\hline \multirow{3}{*}{ C.C. 135.4} & $\begin{array}{l}\text { Deslocamento constante do } \\
\text { operador, pois são utilizadas. }\end{array}$ & & \multirow{3}{*}{$\begin{array}{c}\text { NR 17.1.2.; } \\
\text { NR 17.6.1 ; } \\
\text { NR 17.6.2.a;b;c;d;e;f; } \\
\text { NR17.6.3. a;b;c. }\end{array}$} & \multirow{3}{*}{11} & \multirow{3}{*}{933} & \multirow{3}{*}{194,997} \\
\hline & $\begin{array}{l}\text { O operador sente cansaço nos } \\
\text { membros inferiores ao final do } \\
\text { turno de trabalho. }\end{array}$ & & & & & \\
\hline & $\begin{array}{l}\text { Grande distancia entre as } \\
\text { máquinas no }\end{array}$ & & & & & \\
\hline
\end{tabular}

A combinação da apreciação SPM com a abordagem Fuzzy nos ajuda a determinar o grau de gravidade ocupacional de cada posto de trabalho, ou índice "SIC" (Somatório dos Indicadores de Criticidade Ocupacional), desenvolvido por pesquisadores do laboratório GENTE/COPPE da UFRJ, agora associado ao LABFuzzy da mesma instituição. A tabulação SIC/Fuzzy ou simplesmente "SIC" torna-se, portanto, uma ferramenta dinâmica de suporte a tomada de decisão, construída literalmente pela atuação prática em ergonomia.

Esse processo de priorização foi utilizado pela primeira vez durante os encaminhamentos regulares de um projeto de análise ergonômica em uma grande indústria de calçados brasileira, com várias unidades em todo o país. Naquela ocasião, o grupo de trabalho encarregado das ações teve a necessidade de ajustar a proposta inicial de priorização das ações por critérios mais objetivos do que seriam possíveis de serem apontados por uma ferramenta isolada de priorização.

Tal providência mostrou-se crucial para a correta seleção das oportunidades de melhoria, já que o índice obtido pela aplicação de uma ferramenta de avaliação biomecânica chamada OCRA (OCCHIPINTI, 1998) era uma das variáveis da tabulação e determinava a primeira escala de ações necessárias para implementação das mudanças (Relatório GENTE/UFPB Alpargatas, 2011). Ao atribuirmos a essa variável um peso maior (codificado por cores), a atividade de trabalho correlacionada a essa tabulação se destacava dos outros conjuntos numéricos, facilitando sua identificação e seu posterior tratamento. 0 Quadro 2 ilustra a tabulação operacional de cada índice e o resultado matricial (resumo) de aplicação da Tabulação SIC em uma das fábricas na região nordeste. 
Quadro 2 - Alpargatas Unidade João Pessoa - Oportunidades de Melhorias

\begin{tabular}{|c|c|c|c|c|c|c|c|c|c|c|}
\hline LUGAR & POSTO & 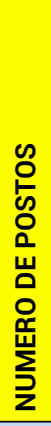 & 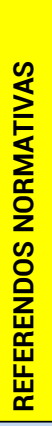 & 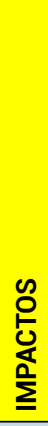 & 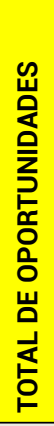 & 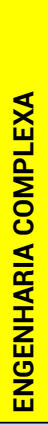 & 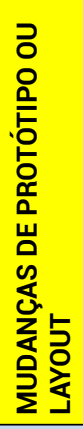 & 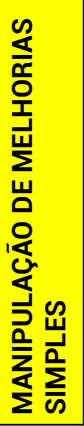 & 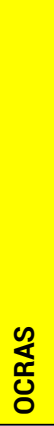 & 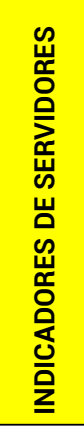 \\
\hline CERTO & LÍDER & 1 & 3 & 3 & 2 & & & 2 & & 0.00 \\
\hline CERTO & OPERADOR & 1 & 3 & 3 & 3 & 1 & & 2 & 1 & 12.00 \\
\hline CERTO & OPERADOR AFA & 1 & 3 & 3 & 3 & 1 & & 2 & 1 & 12.00 \\
\hline \multirow[t]{2}{*}{ CERTO } & REVISADOR & 1 & 3 & 3 & 2 & & & 2 & & 6.00 \\
\hline & & & & & 0 & & & & & \\
\hline ENGENHARIA DE PRODUÇÃO & ANALISTA & 1 & 3 & 2 & 2 & & & 2 & & 6.00 \\
\hline ENGENHARIA DE PRODUÇÃO & MODALISTA & 1 & 3 & 2 & 2 & & & 2 & & 6.00 \\
\hline ENGENHARIA DE PRODUÇÃO & GERENTE & 1 & 3 & 2 & 2 & & & 2 & & 5.00 \\
\hline ENGENHARIA DE PRODUÇÃO & ASSISTENTE & 1 & 4 & 3 & 2 & & & 2 & & 7.00 \\
\hline \multirow[t]{2}{*}{ ENGENHARIA DE PRODUÇÃO } & COSTUREIRA & 1 & 3 & 3 & 2 & & & 2 & & 6.00 \\
\hline & & & & & 0 & & & & & - \\
\hline COSTURA TIMBERLAND & COSTURAR & 1 & 3 & 3 & 3 & 1 & & 2 & 1 & 12.00 \\
\hline COSTURA TIMBERLAND & ESTUFA B & 1 & 3 & 2 & 3 & 1 & & 2 & 1 & 11.00 \\
\hline \multirow[t]{2}{*}{ COSTURA TIMBERLAND } & PRENSA SARL & 1 & 3 & 3 & 2 & & & 2 & & 0.00 \\
\hline & & & & & 0 & & & & & 0 \\
\hline PREPARAÇÃO TIMBERLAND & CARIMBAR & 1 & 3 & 2 & 3 & 1 & & 2 & 1 & 11.00 \\
\hline PREPARAÇÃO TIMBERLAND & CORTAR FITA & 1 & 3 & 3 & 2 & & & 2 & & 6.00 \\
\hline
\end{tabular}

Fonte: dos autores.

Quadro 3 - Resumo da Tabulação dos Índices de Criticidade.

\begin{tabular}{|c|c|c|c|c|c|c|c|c|c|}
\hline & & & & & $\begin{array}{c}\text { Oportunidades de } \\
\text { Melhorias }\end{array}$ & & & \\
\hline Unidades & Postos & Normas & Impacto & TG & EC & PL & IM & Ocra & Sic \\
\hline Campo Grande & 178 & 1004 & 265 & 259 & 30 & 65 & 164 & 3 & 1287 \\
\hline Santa Rita & 116 & 674 & 241 & 256 & 31 & 31 & 166 & 23 & 1053 \\
\hline Totais & 294 & 1674 & 506 & 515 & 61 & 96 & 330 & 26 & 2340 \\
\hline
\end{tabular}

Fonte: dos autores.

Em outro exemplo, uma empresa têxtil no Rio de Janeiro, vinculada a uma marca famosa mundialmente, pode-se comprovar outra importante característica da ferramenta, sua facilidade de aplicação. A flexibilidade de seu emprego, aliada à simplicidade no processo de capacitação para seu emprego, mostrou-se particularmente importante em situações onde os prazos sejam a maior restrição de um projeto. 
Por se tratar de uma ação corrente de demanda de cunho legal - adequação normativa oriunda do Ministério Público - tínhamos uma dupla restrição temporal, imposta em cascata pelas autoridades legais e a própria empresa demandante, além de motivos de confidencialidade legal. Nesse caso, a tabulação foi incorporada ao Plano de Ação (Figura 2), constituindo-se, de uma importante ferramenta de gestão de melhorias e um instrumento de controle das ações de adequação e enquadramento normativo (Relatórios GENTE/COPPE, 2006 a 2012).

Quadro 4 - Extrato de Tabulação SIC

\begin{tabular}{|c|c|c|c|c|c|c|c|}
\hline Posto & Recomendação & $\begin{array}{c}\text { Ação } \\
\text { (A) }\end{array}$ & Enquadramento (E) & GUT (G) & $\begin{array}{c}\text { Ocra } \\
(0)\end{array}$ & SIC & Prazo de Ação \\
\hline \multirow{3}{*}{ Costureira } & $\begin{array}{l}\text { Promover um programa de } \\
\text { pausas e rodízios entre outros } \\
\text { setores para evitar a manutenção } \\
\text { de atividades repetitivas através } \\
\text { da diminuição do estresse } \\
\text { físico sobre a coluna vertebral e } \\
\text { membros superiores. }\end{array}$ & & 8 & \multirow{3}{*}{556} & \multirow{3}{*}{32,79} & \multirow{3}{*}{72,9} & 30 Dias \\
\hline & $\begin{array}{l}\text { Oferecer mobiliário adequado, que } \\
\text { permita regulagens lombares e de } \\
\text { assento e com revestimento que } \\
\text { reduza a compreensão na coluna } \\
\text { e membros inferiores promovendo } \\
\text { melhor conforto durante o } \\
\text { desenvolvimento da tarefa. }\end{array}$ & & \multirow{2}{*}{$\begin{array}{l}\text { NR17.1.1; } \\
\text { NR17.1.2; } \\
\text { NR17.3.1; } \\
\text { NR17.3.2; } \\
\text { NR17.3.3; } \\
\text { NR17.3.5 } \\
\text { NR17.6.2 } \\
\text { NR17.6.3 }\end{array}$} & & & & Até 2 anos \\
\hline & $\begin{array}{l}\text { Estudar junto à engenharia a } \\
\text { possibilidade de desenvolver um } \\
\text { mecanismo de corte de resíduos } \\
\text { acoplado á máquina de costura ou } \\
\text { mesmo ao ponto de trabalho, que } \\
\text { substitua o uso da tesoura. }\end{array}$ & & & & & & Acima de 2 anos \\
\hline
\end{tabular}

Fonte: dos autores.

A tabulação SIC, portanto, ajuda a determinar o grau de risco ergonômico de cada posto de trabaIho, a partir da combinação de variáveis de diferentes matrizes (Matriz Observacional, Enquadramento Normativo e demais ferramentas de apreciação e de avaliação global das situações de trabalho). Como pode ser notado, a modelagem Fuzzy não altera significativamente os resultados, porém oferece uma maior precisão ao computar variáveis comportamentais e linguísticas, o que também aumenta a credibilidade ao método. Em sua forma simplificada, portanto, o índice SIC/Fuzzy é dado por:

SIC $=K$ [Constante de Fatoração] * F[ Modelagem Fuzzy] * [Matriz GUT] * [Numero de desconformidades ]

Uma vez isoladas as alternativas acima de uma determinada escala de severidade - ou gravidade - do ponto de vista ocupacional, o plano de ação integrante de um documento maior da ação ergonômica (laudo, relatório, etc.) deverá contemplar uma ordem de priorização partindo dos níveis escolhidos. Em geral, os níveis codificados na cor roxa são aqueles que apresentam os maiores riscos associados para o trabalhador, porém isso dependerá dos critérios definidos pela equipe de ergonomia em ação conjunta com os usuários de um sistema de trabalho. 
A teoria dos conjuntos fuzzy contribui para auxiliar na interpretação do modo aproximado de raciocínio humano, levando-o para um formato numérico e obtendo-se respostas em ambiente de incerteza que servem de subsídio à tomada de decisão. Nesse trabalho desenvolveu-se um modelo fuzzy, utilizando fatores provocadores de criticidade, para verificar os processos de trabalho, as ações e fornecer elementos, a partir de uma priorização, para a formulação de diagnóstico e recomendações.

A teoria dos conjuntos fuzzy, introduzida em 1965 (ZADEH, 1965), é utilizada para representar modelos de julgamentos humanos, que possuem um papel essencial na habilidade humana de decidir racionalmente, em ambientes de incertezas e imprecisões. Essa teoria procura solucionar o problema do tratamento de informações de caráter impreciso, não passíveis de processamento por meio da lógica convencional. Esse novo conceito deu origem à lógica fuzzy. Ao contrário da lógica convencional (binária ou booleana), a lógica fuzzy é multivariada e, em vez de um elemento pertencer $100 \%$ a um conjunto ou uma proposição ser somente verdadeira ou falsa, é possível trabalhar com informações parcialmente verdadeiras ou parcialmente falsas.

Em razão disso, a lógica fuzzy é uma técnica que fornece instrumentos para análise de informações imprecisas, incompletas e até não confiáveis, que representam conceitos subjetivos, tais como as opiniões e os sentimentos. Na teoria dos conjuntos fuzzy existe um grau de pertinência de cada elemento a um determinado conjunto. Não existe uma fronteira bem definida para decidirmos quando um elemento pertence ou não pertence ao respectivo conjunto. Desta forma, um conjunto fuzzy pode ser representado por um conjunto de pares ordenados, em que o primeiro elemento é $x \in X$, e o segundo, $\mu \mathrm{A}(\mathrm{x})$, é o grau de pertinência ou função de pertinência de $\mathrm{x}$ em $A$, que mapeia $X$ para o espaço de pertinência $M$. Quando $M$ contem apenas os pontos 0 e 1, A é não fuzzy. Desta forma, temos como resultado a Equação 1:

$A=\{(x, \mu A(x)) \mid x \in X\}$

As informações nebulosas podem ser modeladas por números fuzzy. Os números fuzzy são constituídos por conjuntos fuzzy, definidos em universos de discurso discretos ou contínuos, que permitem a quantificação da incerteza e imprecisão associada a uma informação (HSU e CHEN, 1996; LIANG e WANG, 1991). Dentre as diversas formas de números fuzzy, o número fuzzy triangular é o mais utilizado (PEDRYCZ, 1994). Este número fuzzy é representado por três pontos e pode ser expresso, por exemplo, por $A=(a 1, a 2, a 3)$, conforme ilustrado na Figura 1. Esta representação é interpretada como funções de pertinência (Equação 2). 
Figura 1 - Ilustração gráfica do número fuzzy $A=\left(a_{1}, a_{2}, a_{3}\right)$.

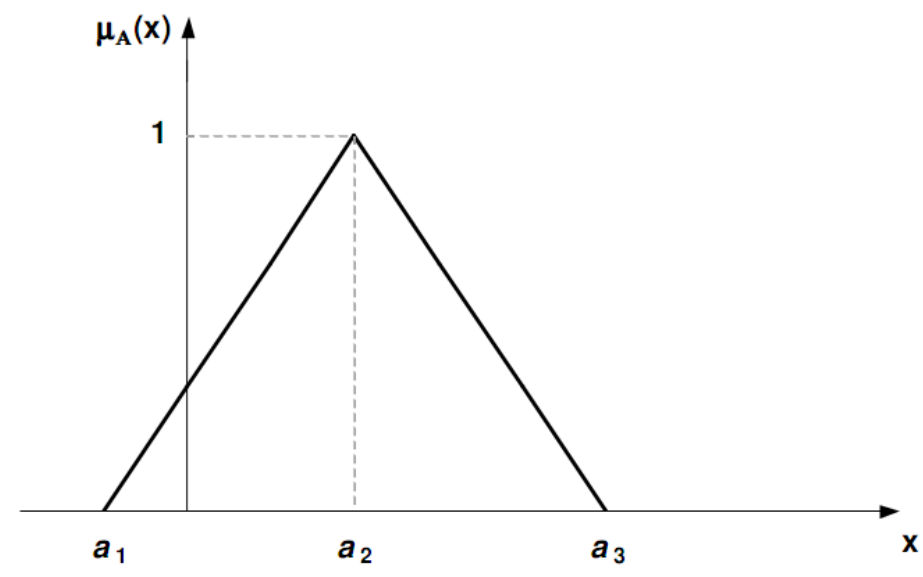

Fonte: dos autores

Equação 2:

$\mu_{A}(x)= \begin{cases}0, & x<a_{1} \\ \frac{x-a_{1}}{a_{2}-a_{1}}, & a_{1} \leq x \leq a_{2} \\ \frac{a_{3}-x}{a_{3}-a_{2}}, & a_{2} \leq x \leq a_{3} \\ 0, & x>a_{3}\end{cases}$

O parâmetro "a2" apresenta o grau máximo de pertinência, ou seja, $\mu \mathrm{A}(\mathrm{x})=1$. Esse é o maior valor possível de uma avaliação (opinião) feita por um indivíduo. Os parâmetros "a1" e "a3" representam o limite inferior e superior da área de avaliação, a qual representa a nebulosidade da avaliação (opinião) feita pelo indivíduo.

A seguir são apresentadas as etapas do modelo fuzzy para priorização por criticidade. Este modelo é uma versão adaptada do modelo fuzzy de avaliação da resiliência organizacional proposto por Grecco (2012).

\subsection{Etapa de Definição dos fatores (indicadores) causadores de criticidade.}

Nessa etapa são definidos os fatores que, de forma ponderada e combinada, irão compor o índice final de criticidade do posto ou atividade de trabalho, que por sua vez ajudará na elaboração do plano de ação para dar conta das inadequações verificadas. No caso do estudo principal onde a modelagem fuzzy foi aplicada, o quadro de fatores pode ser observado no Quadro 5. 
Quadro 5 - Indicadores de Segurança, Saúde e Qualidade Operacional

\begin{tabular}{|c|c|}
\hline Dimensões & Indicadores \\
\hline Segurança & $\begin{array}{l}\text { 1.1 Recursos humanos } \\
\text { 1.2 Treinamentos } \\
\text { 1.3 Comunicação } \\
\text { 1.4 Adaptações locais } \\
\text { 1.5 Conteúdo das documentações } \\
\text { 1.6 Disponibilidade das documentações } \\
\text { 1.7 Capacidade de controlar situações imprevistas } \\
\text { 1.8 Informações de segurança } \\
\text { 1.9 Carga de trabalho } \\
\text { 1.10 Trabalho em equipe } \\
\text { 1.11 Compreensão das limitações } \\
\text { 1.12 Manutenção preventiva } \\
\text { 1.13 Identificação de riscos } \\
\text { 1.14 Equipamentos de segurança } \\
\text { 1.15 Sistemas de alarmes }\end{array}$ \\
\hline $\begin{array}{l}\text { Conforto e } \\
\text { Higiene do Trabalho }\end{array}$ & $\begin{array}{l}\text { 2.1 Ruído } \\
2.2 \text { Iluminação } \\
2.3 \text { Qualidade do ar } \\
2.4 \text { Condições térmicas } \\
2.5 \text { Limpeza do local } \\
2.6 \text { Ventilação } \\
2.7 \text { Contato com produtos perigosos } \\
2.8 \text { Circulação do setor } \\
2.9 \text { Trabalho em pé } \\
2.10 \text { Espaço interno e Layout } \\
2.11 \text { Mobiliário } \\
2.12 \text { Esforço corporal }\end{array}$ \\
\hline Qualidade e Sustentabilidade & $\begin{array}{l}\text { 3.1 Clientes satisfeitos } \\
\text { 3.2 Falhas na produção } \\
\text { 3.3 Entregas no prazo } \\
\text { 3.4 Desperdícios } \\
\text { 3.5 Tempo de produção } \\
\text { 3.6 Habilitação para o serviço } \\
\text { 3.7 Recursos materiais } \\
\text { 3.8 Recursos naturais } \\
\text { 3.9 Impactos Ambientais }\end{array}$ \\
\hline
\end{tabular}

Fonte: dos autores. 


\subsection{Etapa de Determinação dos critérios de Avaliação dos Indicadores.}

Uma vez definidos os indicadores de QSMS (quadro 1), devem ser estabelecidos os fatores de atenuação e respectivos critérios de avaliação de cada indicador. Isso permitirá na sequencia a construção da matriz de priorização. Após a definição dos critérios de avaliação, o modelo pressupõe a aplicação de um questionário, que tem por objetivo determinar o nível de importância de indicadores relacionados a segurança, conforto, higiene do trabalho, qualidade e sustentabilidade, para avaliação da criticidade na apreciação dos postos de trabalho operacionais.

O modelo de priorização Fuzzy tende a reduzir a subjetividade dos resultados dos diversos instrumentos de verificação, como questionários e check lists (listas de verificação), já que a qualidade da pesquisa depende da legitimidade e precisão na coleta de dados e do nível de transparência das opiniões interpretadas pelos especialistas.

\section{DESENVOLVIMENTO DO ESTUDO}

O estudo-piloto que utiliza o modelo proposto de matriz envolve observações sistematizadas em canteiros de obras de engenharia, onde foram registrados dados relevantes para caracterização das diversas atividades produtivas.

A matemática fuzzy serve para, no caso específico do modelo proposto, agregar as diversas e diferentes opiniões - e os diferentes pesos que possam ser atribuídos - de forma a se elaborar uma matriz de hierarquização dos indicadores. São atribuídas as devidas "importâncias", segundo a escala apresentada em tabelas contendo índices de satisfação ou atendimento de conformidade, aos respectivos indicadores de desempenho. Assim, Para a primeira relação de indicadores, utiliza-se o quadro de níveis de importância (Quadro 6), que cada avaliador preenche de acordo com sua percepção sobre o contexto observado.

Quadro 6 - Escala de Importância

\begin{tabular}{|c|l|}
\hline Importância & Explicação \\
\hline NI & O indicador apresentado não é importante. \\
\hline PI & O indicador apresentado é pouco importante. \\
\hline I & O indicador apresentado é importante. \\
\hline MI & O indicador apresentado é muito importante. \\
\hline
\end{tabular}

Fonte: dos autores.

A codificação, que deve ser amplamente negociada, precisa ser então aplicada aos diferentes indicadores de condições do posto ou atividade. No caso do estudo em tela, foram atribuídos os seguintes valores, conforme o Quadro 7. 
Quadro 7 - Classificação da Escala de Importância

\begin{tabular}{|c|c|}
\hline \multicolumn{2}{|l|}{ Segurança } \\
\hline Indicadores & Importância \\
\hline $\begin{array}{l}\text { Recursos Humanos: Avalia se a quantidade de trabalhadores no setor é suficiente para garantir a execução } \\
\text { segura das tarefas. }\end{array}$ & Ml \\
\hline $\begin{array}{l}\text { Treinamentos: Avalia se os treinamentos adequados às atividades dos trabalhadores são frequentemente } \\
\text { oferecidos e incentivados pela chefia. }\end{array}$ & I \\
\hline $\begin{array}{l}\text { Comunicação: Avalia se os mecanismos de comunicação são eficientes para divulgação de informações sobre } \\
\text { incidentes e informações relevantes ao trabalho. }\end{array}$ & I \\
\hline $\begin{array}{l}\text { Adaptações locais: Avalia se as adaptações das tarefas às condições locais são efetuadas conhecendo seus } \\
\text { efeitos sobre a segurança. }\end{array}$ & Ml \\
\hline Conteúdo das documentações: Avalia se os procedimentos, instruções ou documentações são atualizados e de fácil compreensão. & $\mathrm{Ml}$ \\
\hline $\begin{array}{l}\text { Disponibilidade das documentações: Avalia se os procedimentos, instruções ou documentações estão } \\
\text { disponíveis para as pessoas quando necessário. }\end{array}$ & MI \\
\hline $\begin{array}{l}\text { Capacidade de controlar situações imprevistas: Avalia se as pessoas são treinadas para controlar situações } \\
\text { novas ou imprevistas na ausência de procedimentos ou instruções. }\end{array}$ & I \\
\hline $\begin{array}{l}\text { Informações de segurança: Avalia se as pessoas são informadas sobre orientações de segurança e assuntos que } \\
\text { interferem na execução de suas atividades. }\end{array}$ & MI \\
\hline Carga de trabalho: Avalia se o conteúdo e o volume de trabalho não colocam em risco a saúde das pessoas. & MI \\
\hline Trabalho em equipe: Avalia se existe um bom relacionamento entre as equipes (grupos) de trabalho. & $\mathrm{Ml}$ \\
\hline $\begin{array}{l}\text { Compreensão das limitações: Avalia se as pessoas têm percepção e consciência das condições técnicas dos } \\
\text { recursos materiais e limitações dos procedimentos e documentações. }\end{array}$ & I \\
\hline Manutenção preventiva: Avalia se existe um programa de manutenção preventiva dos equipamentos. & $\mathrm{Ml}$ \\
\hline $\begin{array}{l}\text { Identificação de riscos: Avalia se existem medidas proativas no setor para identificar novos riscos (avaliação de } \\
\text { riscos), utilizando seus resultados no desenvolvimento de políticas, procedimentos ou práticas (ações corretivas). }\end{array}$ & I \\
\hline Equipamentos de segurança: Avalia se os trabalhadores executam suas tarefas com equipamentos de segurança adequados. & $\mathrm{I}$ \\
\hline Sistemas de alarme: Avalia se existe um sistema de alarmes no local para respostas às emergências. & $\mathrm{PI}$ \\
\hline \multicolumn{2}{|l|}{ Conforto e Higiene do Trabalho } \\
\hline Indicadores & Importância \\
\hline Ruído: Avalia se o nível de ruído no local de trabalho não afeta o bem-estar dos trabalhadores. & $\mathrm{Ml}$ \\
\hline Iluminação: Avalia se a iluminação no local de trabalho é adequada para execução das atividades. & $\mathrm{Ml}$ \\
\hline Qualidade do ar: Avalia se a qualidade do ar no local de trabalho não afeta o bem-estar dos trabalhadores. & MI \\
\hline Condições térmicas: Avalia se as condições térmicas (temperatura e umidade) são adequadas para execução das atividades. & $\mathrm{Ml}$ \\
\hline Limpeza do local: Avalia se a limpeza do local de trabalho é adequada para execução das atividades. & 1 \\
\hline Ventilação: Avalia se a ventilação do local de trabalho é adequada para execução das atividades. & 1 \\
\hline $\begin{array}{l}\text { Contato com produtos perigosos: Avalia se o manuseio de produtos perigosos é feito de maneira a garantir a } \\
\text { segurança dos trabalhadores. }\end{array}$ & Ml \\
\hline $\begin{array}{l}\text { Circulação: Avalia se as vias de acesso e de circulação do local de trabalho estão em condições adequadas para } \\
\text { a movimentação dos trabalhadores e de materiais (cargas). }\end{array}$ & I \\
\hline Trabalho em pé: Avalia se os trabalhos realizados em pé não são envolvem longos períodos e não causam fadiga. & $\mathrm{PI}$ \\
\hline Mobiliário: Avalia se o mobiliário (armários, bancadas, assentos) não apresenta deficiência. & $\mathrm{NI}$ \\
\hline $\begin{array}{l}\text { Esforço corporal (físico): Avalia se os trabalhadores não são submetidos a esforços excessivos, como por } \\
\text { exemplo, carregamento manual de peso, número elevado de tarefas com curto ciclo de duração, esforços } \\
\text { excessivos no acionamento de máquinas e dispositivos. }\end{array}$ & Ml \\
\hline \multicolumn{2}{|l|}{ Qualidade e Produtividade } \\
\hline Indicadores & Importância \\
\hline Clientes satisfeitos: Avalia se o trabalho produzido atende aos clientes. & MI \\
\hline Falhas na produção: Avalia se as falhas no processo produtivo são identificadas e corrigidas, não afetando a qualidade. & MI \\
\hline Entregas no prazo: Avalia se as unidades produzidas (trabalhos produzidos) são entregues (feitas) no prazo. & $\mathrm{Ml}$ \\
\hline Desperdícios: Avalia se os desperdícios de produção são poucos, não gerando custos financeiros e operacionais. & $\mathrm{Ml}$ \\
\hline $\begin{array}{l}\text { Tempo de produção: Avalia se o tempo médio de produção é adequado, não interferindo na carga de trabalho e na } \\
\text { qualidade dos produtos. }\end{array}$ & MI \\
\hline Habilitação para o serviço: Avalia se as exigências das tarefas estão alinhadas com as habilidades das pessoas. & $\mathrm{Ml}$ \\
\hline $\begin{array}{l}\text { Recursos materiais: Avalia se a qualidade e a disponibilidade dos recursos materiais (equipamentos, ferramentas, } \\
\text { matéria-prima) são adequadas para a execução das atividades. }\end{array}$ & MI \\
\hline
\end{tabular}


O mesmo procedimento adotado para as atividades de natureza operacional foi então empregado para postos administrativos e ampliado de acordo com o propósito de cada momento da avaliação.

\section{RESULTADOS PRELIMINARES}

Os resultados das diversas experiências de "fuzzyficação" das variáveis e indicadores de desempenho utilizadas no processo de decisório mostraram que a alternativa de incorporação da lógica fuzzy ainda precisa ser vista com responsável cautela. Se por um lado existe a possibilidade de ajustar os cenários observáveis às necessidades de melhorias nos locais de trabalho, por outro o modelo depende de grande esforço de gestão de forma a impedir que julgamentos imparciais possam comprometer as ações de melhoria que vêm à tona na esteira dos processos de ação ergonômica ou qualquer processo de melhoria contínua.

Para efeito de ilustração, e salvaguardando-se o princípio de confidencialidade, isolou-se um dos indicadores de um dos estudos citados para uma melhor descrição do processo. 0 resultado da avaliação do indicador "Recursos Materiais", relativo ao princípio da engenharia de resiliência "Comprometimento da Alta Direção", é dado por N, calculado pela Equação 3, que também é um número fuzzy triangular.

$N=\sum_{i=l}^{n}\left(C C E_{i} \cdot n_{i}\right)$

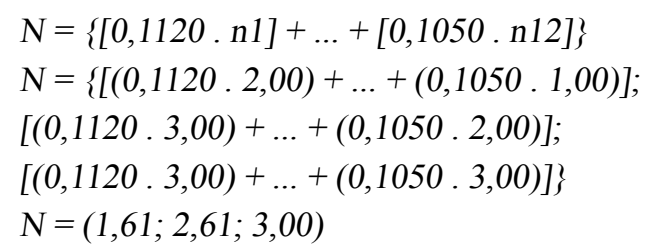

Este número fuzzy $\mathrm{N}$ é representado na Figura 2 a seguir.

Figura 2 - Função de pertinência do indicador

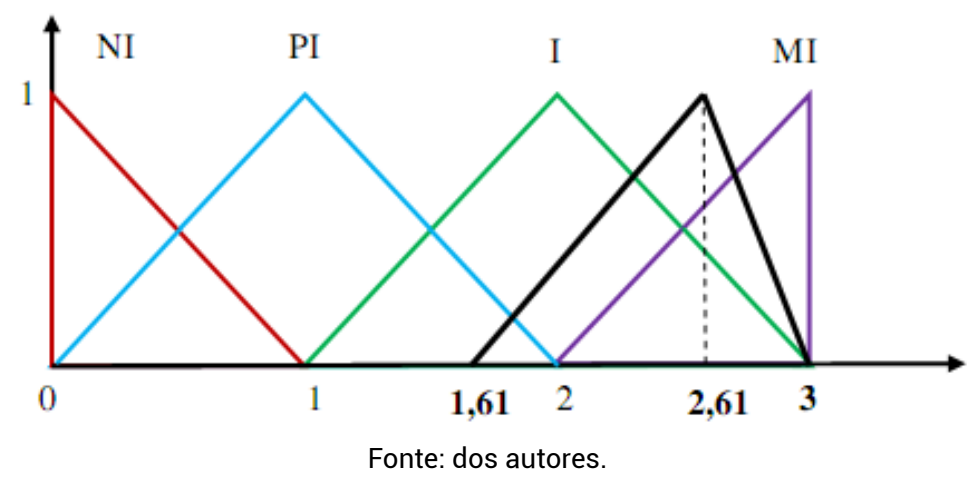

Nota-se que o resultado indicado no gráfico nada mais é que uma ponderação de possibilidades, o que mostra relação com os modelos preliminares de fatoração utilizados nos estudos iniciais onde 
a Matriz de Priorização SIC foi utilizada. A Tabela 1 a seguir mostra o resultado da avaliação de todos os indicadores utilizados nesta aplicação.

Tabela 1 - Indicadores e Variáveis "fuzzyficadas"

\begin{tabular}{|c|c|c|c|c|}
\hline \multirow[t]{2}{*}{ Indicadores } & \multicolumn{4}{|c|}{ Número fuzzy } \\
\hline & a & b & c & GII \\
\hline \multicolumn{5}{|l|}{ Comprometimento da Alta Direção } \\
\hline 1.1 Recursos Humanos & 1,51 & 2,51 & 3,00 & 0,893 \\
\hline 1.2 Recursos Materiais & 1,61 & 2,61 & 3,00 & 0,929 \\
\hline 1.3 Compromisso com a Segurança & 1,51 & 2,51 & 3,00 & 0,893 \\
\hline 1.4 Política de Segurança & 1,21 & 2,21 & 3,00 & 0,786 \\
\hline 1.5 Objetivos de Segurança & 1,47 & 2,47 & 2,97 & 0,879 \\
\hline 1.6 Treinamentos & 1,81 & 2,81 & 3,00 & 1,000 \\
\hline 1.7 Identificação de Competências & 1,06 & 2,06 & 2,89 & 0,733 \\
\hline \multicolumn{5}{|l|}{ Aprendizagem } \\
\hline 2.1 Comunicação & 1,20 & 2,20 & 2,89 & 0,827 \\
\hline 2.2 Conteúdo das Informações & 1,15 & 2,15 & 2,95 & 0,808 \\
\hline 2.3 Execução das Tarefas & 1,49 & 2,49 & 2,85 & 0,936 \\
\hline 2.4 Práticas Reais de Trabalho & 1,35 & 2,35 & 2,85 & 0,883 \\
\hline 2.5 Adaptações Locais & 1,51 & 2,51 & 3,00 & 0,944 \\
\hline 2.6 Conteúdo das Documentações & 1,61 & 2,61 & 3,00 & 0,981 \\
\hline 2.7 Disponibilidade das Documentações & 1,37 & 2,37 & 2,95 & 0,891 \\
\hline 2.8 Investigações de Incidentes & 1,66 & 2,66 & 3,00 & 1,000 \\
\hline 2.9 Responsabilidade das Investigações de Incidentes & 1,04 & 2,04 & 3,00 & 0,767 \\
\hline \multicolumn{5}{|l|}{ Flexibilidade } \\
\hline 3.1 Capacidade de Controlar Situações Imprevistas & 1,65 & 2,65 & 2,92 & 0,964 \\
\hline 3.2 Flexibilidade das Atividades & 1,29 & 2,29 & 2,85 & 0,833 \\
\hline 3.3 Reconhecimento Profissional & 1,23 & 2,23 & 3,00 & 0,811 \\
\hline 3.4 Limites de Trabalho Seguro & 1,42 & 2,42 & 2,95 & 0,880 \\
\hline 3.5 Relatos das Adaptações & 1,63 & 2,63 & 3,00 & 0,956 \\
\hline 3.6 Incorporação de Adaptações & 1,75 & 2,75 & 3,00 & 1,000 \\
\hline \multicolumn{5}{|l|}{ Consciência } \\
\hline 4.1 Relatos de Problemas & 1,65 & 2,65 & 2,89 & 1,000 \\
\hline 4.2 Informações de Segurança & 1,60 & 2,60 & 3,00 & 0,981 \\
\hline 4.3 Mecanismos de Comunicação & 1,16 & 2,16 & 2,81 & 0,815 \\
\hline 4.4 Disponibilidade para Substituições & 0,84 & 1,84 & 2,53 & 0,694 \\
\hline 4.5 Carga de Trabalho & 1,28 & 2,28 & 3,00 & 0,860 \\
\hline 4.6 Trabalho em Equipe & 1,36 & 2,36 & 2,95 & 0,891 \\
\hline 4.7 Tarefas e Habilidades das Pessoas & 1,28 & 2,28 & 3,00 & 0,860 \\
\hline 4.8 Compreensão das Limitações & 1,49 & 2,49 & 2,97 & 0,940 \\
\hline 4.9 Manutenção Preventiva & 1,40 & 2,40 & 3,00 & 0,913 \\
\hline 4.10 Identificação de Perigos & 1,42 & 2,42 & 3,00 & 0,913 \\
\hline \multicolumn{5}{|l|}{ Cultura de Justiça } \\
\hline 5.1 Relato de Desvios/ Erros & 1,28 & 2,28 & 2,93 & 0,870 \\
\hline 5.2 Entendimento dos Erros & 1,11 & 2,08 & 2,85 & 0,794 \\
\hline 5.3 Percepção dos Erros & 1,23 & 2,23 & 2,93 & 0,851 \\
\hline 5.4 Ações não Punitivas & 1,11 & 2,02 & 2,79 & 0,771 \\
\hline 5.5 Opinião da Equipe nas Investigações & 1,62 & 2,62 & 2,98 & 1,000 \\
\hline \multicolumn{5}{|l|}{ Preparação para os Problemas } \\
\hline 6.1 Plano de Resposta à Emergência & 1,49 & 2,49 & 3,00 & 0,883 \\
\hline 6.2 Identificação dos Riscos & 1,73 & 2,73 & 3,00 & 0,968 \\
\hline 6.3 Equipamentos de Segurança & 1,56 & 2,56 & 3,00 & 0,908 \\
\hline 6.4 Sistemas de Alarme & 1,60 & 2,60 & 3,00 & 0,922 \\
\hline 6.5 Procedimentos Proativos & 1,82 & 2,82 & 3,00 & 1,000 \\
\hline 6.6 Treinamento de Resposta à Emergência & 1,53 & 2,53 & 3,00 & 0,897 \\
\hline
\end{tabular}




\section{CONCLUSÃO}

Como todo e qualquer construto matemático, o modelo de priorização proposto é uma aproximação da realidade com um nível de precisão suficiente para auxiliar o processo decisório e não para precisar a decisão correta. No caso do modelo, há uma orientação assumida para parâmetros de centralidade (médias, equilíbrios e outros pontos de convergência clássica). Sem a utilização de uma ferramenta de priorização adequada não teria sido possível selecionar os postos de trabalho dentro de critérios coerentes, o que poderia ter levado a uma conclusão errônea em relação ao ponto principal do estudo que, no caso do estudo-piloto, correspondia a validação da exigência de alocação de tempos de reposição de fadiga.

Os resultados positivos obtidos nos outros casos apresentados estabelecem uma suficiente relação de causa-feito entre a aplicação da ferramenta e a sua viabilização enquanto instrumento de planejamento e gestão, com potencial evidente de extrapolação para além dos domínios da Ergonomia. Ao se alterar a forma de priorização do GUT (fatoração com média ponderada) pela modelagem Fuzzy, percebemos imediatamente a redução da importância do peso de certas variáveis comportamentais de análise e interpretação dos dados de área. Como exemplo, a modelagem permite equilibrar níveis de capacitação dos analistas, minimizando a possibilidade de subdimensionamento dos impactos ocupacionais.

Complementando, a consolidação como ferramenta de ação ergonômica se condiciona à sua replicação em estudos de natureza similar. Isso é esperado com a disseminação dos resultados preliminares, garantindo-se assim sua otimização. A prática tenderá a calibrar de forma contínua os atributos utilizados na modelagem fuzzy, trazendo então sua consolidação enquanto conceito e ferramenta de processo. 


\section{REFERÊNCIAS}

BOUYSSOU, D., et al. (Eds), Decision Making Process, Concepts and Methods, Hoboken, John Wiley \& Sons, 2010.

GENTE/COPPE, Relatórios de Projetos, Laboratório GENTE/COPPE/UFRJ, 2006-2012.

GENTE/COPPE/UFPB, Relatório Alpargatas Final, GENTE/COPPE/UFPB, 2010.

GRANDJEAN, E., , Manual de ergonomia: adaptando o trabalho ao homem, $4^{\mathrm{a}}$ ed. Porto Alegre: Ed. Bookman, 1998

GRECCO, C. H. S., VIDAL, M. C. R, CARVALHO, P. V. R., Using Fuzzy Set Theory to Model Resilience in Safe-Critical Organizations. In: AHRAM, T. Z., KARWOWSKI, W. (Org.). Advances in Physical Ergonomics and Safety. $1^{\text {a }}$ ed. v. 1, 78-87. USA: Taylor \& Francis, 2012.

HENDRICK, H, Handbook of Human Factors and Ergonomics Methods, London: Taylor \& Francis, 2004.

HODGKINSON, G., STARBUCK, W., (Eds), The Oxford Handbook of Organizational Decision Making, Oxford, Oxford University Press, 2008.

HSU, H. M., CHEN, C. T., Aggregation of fuzzy opinions under group decision making. Fuzzy Sets and Systems 79, $279-285,1996$.

LIANG, G. S., WANG, M. J., A fuzzy multi-criteria decision-making method for facility site selection. Int. J. Prod. Res., v. 29, n. 11, 2313-2330, 1991.

MOREIRA, S.B., Fatores humanos no Poder Aeroespacial: aspectos de ergonomia física, UNIFA Universidade da Força Aérea, 2010.

MURRELL, K., Human performance in industry, New York: Reinhold Publishing, 1965.

OCCHIPINTI, E. OCRA, a concise index for the assessment of exposure to repetitive movements of the upper limbs, Ergonomics,41, 1290-1331, 1998.

PEDRYCZ, W., Why triangular membership functions? Fuzzy Sets and Systems 64, 21-30, 1994.

SANTOS, M., VIDAL, M., A Ergonomia de Concepção na Prevenção de Inadequações no Ambiente de Trabalho Construído. Ação Ergonômica, v. 3, p. 24-30, 2008.

SANTOS, M., VIDAL, M., MOREIRA, S., The RFad Method - a new fatigue recovery time assessment for industrial activities, Journal of Prevention, Assessment and Rehabilitation, Volume 41, Supplement 1 / 2012, 1656-1663, 2012.

ZADEH, L. A., Fuzzy Sets, Information Control 8, 338 -353, 1965.

Carayon, P. (1993), Job Design and Job Stress in Office Workers, Ergonomics, 36, May, p. 463-477.

Murrell, K., Bhatia, N., (1969). An industrial experiment in organized rest pauses. Human Factors 11 (2), 167-174. 
Moreira, S. (2010), Notas de Aula, Universidade da Força Aérea (UNIFA), Rio de Janeiro.

Santos, M. (2010) Ergonomic Pattern Mapping - A proposed method for participatory design processes in the workplace. Applied Human Factors and Ergonomics (AHFE) Conference. 